\title{
Guest editorial: Special issue on distributed robotics- from fundamentals to applications
}

\author{
Roderich Groß ${ }^{1} \cdot$ Andreas Kolling $^{2} \cdot$ Spring Berman $^{3} \cdot$ Alcherio Martinoli $^{4} \cdot$ Emilio Frazzoli $^{5} \cdot$ Fumitoshi Matsuno $^{6}$
}

Published online: 22 September 2018

(c) Springer Science+Business Media, LLC, part of Springer Nature 2018

Distributed robotics is an interdisciplinary and rapidly growing area, combining research in computer science, communication and control systems, and electrical and mechanical engineering. Distributed robotic systems can autonomously solve complex problems while operating in highly unstructured real-world environments. They are expected to play a major role in addressing future societal needs, for example, by improving environmental impact assessment, food supply, transportation, manufacturing, security, and emergency and rescue services.

This special issue aims at presenting state-of-the-art research in distributed robotics, leading to advances in technologies, algorithms, system architectures, and applications.

The special issue was preceded by the 13th International Symposium on Distributed Autonomous Robotic Systems (DARS 2016), held at the Natural History Museum, London, UK (November 6-9, 2016) (Groß 2018). Following an open call for papers, 71 submissions to the special issue were received. After a rigorous peer-review, 17 papers were

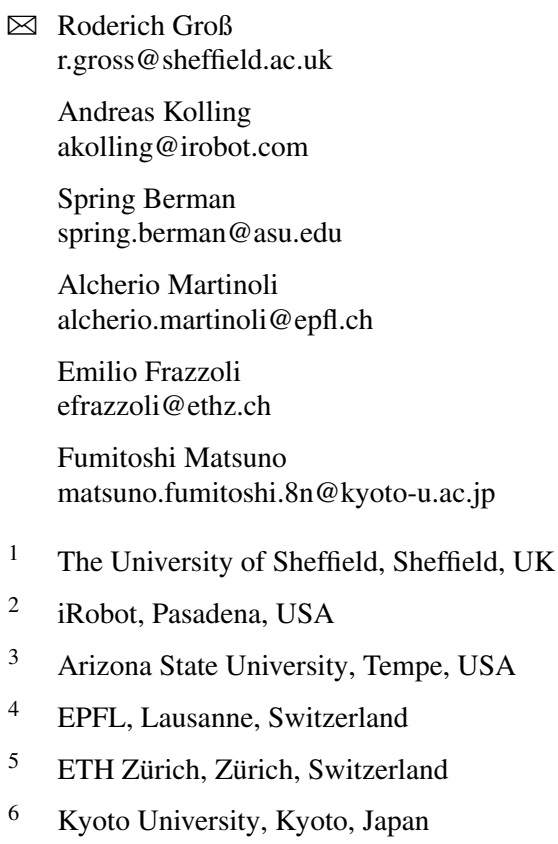

selected for inclusion in this special issue, corresponding to an acceptance rate of $23.9 \%$.

These 17 papers report recent advances in distributed robotics-from fundamentals to applications. The individual contributions are summarized in alphabetic order below.

A distributed control and parameter estimation protocol with prescribed performance for homogeneous Lagrangian multi-agent systems (Bechlioulis, Demetriou, and Kyriakopoulos)

This paper presents a leader-follower scheme for formation control of uncertain homogeneous Lagrangian nonlinear multi-agent systems with a directed communication topology. A distributed adaptive control protocol is used to synchronize the followers' parameter estimates and accurately achieve a rigid formation arbitrarily quickly. The proposed distributed control and parameter estimation protocol is validated through simulation studies on spacecraft attitude control.

ALAN: adaptive learning for multi-agent navigation

(Godoy et al.)

This paper bridges the gap between local and global motion coordination for a multi-agent system by accounting for the influence of local velocity choices on global state. Global coordination is improved sequentially through a sum of rewards in a multi-armed bandit formulation.

Bound to help: cooperative manipulation of objects via compliant, unactuated tails (Kim and Shell)

This paper examines a group of robots with unactuated, compliant "tails", allowing a robot to tow a single object on its own, or, by physically linking with another robot, to tow multiple objects at once. The associated planning problem for Multi-Object Collection via Cooperative Towing is proven to be NP-hard. Exact and heuristic solutions are presented and validated with teams of physical and simulated robots. 
Decentralized navigation method for a robotic swarm with nonhomogeneous abilities (Yoshimoto et al.)

This paper considers swarm motion coordination with a leader in two-dimensional environments for swarms that have heterogenous capabilities in terms of sensor range, velocity and acceleration. In the proposed solution, the followers choose robots to be (unidirectionally) connected with, thereby maintaining the connectivity of the swarm. The control strategy is analyzed theoretically and tested in numerical simulations and experiments with real robots.

Decentralized planning and control for UAV-UGV cooperative teams (Arbanas et al.)

This paper presents a decentralized hierarchical planning and coordination framework for cooperative multi-robot teams composed of unmanned aerial vehicles (UAVs) and unmanned ground vehicles (UGVs). The framework includes high-accuracy motion planning for each robot and ad-hoc mission planning, and it can construct and coordinate feasible team plans in real-time for any input map of the environment. Experiments with a manipulator-equipped UAV and a lightweight UGV in a parcel transportation scenario show that the computed plans are energy-efficient and effective for different environments.

Designing a quasi-spherical module for a huge modular robot to create programmable matter (Piranda and Bourgeois)

This paper presents a quasi-spherical micro-robotic module, called a "catom" in the context of the Claytronics project, that satisfies the requirements for implementing programmable matter. The paper also proposes methods for manufacturing the modules and rules that can be used to program self-reconfiguration algorithms for the modules. Physical modules are fabricated to verify that the proposed movements and latching can be achieved.

Distributed camouflage for swarm robotics and smart materials (Li, Klingner, and Correll)

This paper presents a distributed robotic system that can camouflage itself against its environment. Each robot senses the color and brightness of its local surroundings and interacts only with its neighbors to recognize the environmental pattern and create a suitable camouflage. The system is implemented and experimentally tested on a group of 64 Droplet robots.

Distributed inference-based multi-robot exploration (Smith and Hollinger)

This paper proposes to use observed and inferred structures in maps of unknown environments to compute expected information gain for multi-robot exploration. Information gain and expected travel costs are considered in local auctions to allocate tasks to robots. A comparison against greedy frontier-based and information-based exploration shows a $13.15 \%$ reduction in the joint exploration path lengths.

Distributed system of autonomous buoys for scalable deployment and monitoring of large waterbodies (Zoss et al.)

This paper showcases a distributed system of 50 small, low-cost, self-propelled buoys that are deployable in open waters for monitoring. Flocking, navigation, and area coverage behaviors are tested and shown to be robust to loss of individual buoys.

Dynamic teams of robots as ad hoc distributed computers: reducing the complexity of multi-robot motion planning via subspace selection (Otte and Correll)

This paper presents an approach for a group of robots to collectively plan their motions by dynamically grouping robots that may collide into teams. A heuristic based on a tile-like pebble motion game is used to further constrain the motion of robots within teams. Results from simulations and real-world experiments are presented to validate the approach.

Information-sharing and decision-making in networks of radiation detectors (Yadav et al.)

This paper addresses a scenario in which a sensor network observes a time-inhomogeneous Poisson process and, within a fixed amount of time, must decide between two hypotheses concerning the intensity of the process. The paper demonstrates that network centrality concepts can be used to determine how to route sensor information in the network and identify the sensor that is best positioned to make a decision for the network. The analysis is illustrated with examples of networked radiation detection, both with simulated data and actual radiation measurements.

Limited range spatial load balancing in non-convex environments using sampling-based motion planners (Boardman, Harden, and Martínez)

This paper defines a load-balancing problem for spatial tasks executed by agents with limited range and differential constraints in non-convex environments. The proposed solution approximates an optimal partition with a probabilistic roadmap and a distributed coordination algorithm is shown to converge to the approximated solution. Additional solutions are evaluated in simulations.

Multi robot collision avoidance in a shared workspace (Claes and Tuyls) 
This paper presents a decentralized navigation algorithm, based on the velocity obstacles paradigm, that implements collision avoidance for unmapped static and dynamic obstacles and can be applied in workspaces shared by humans and robots. The approach uses adaptive Monte Carlo localization to estimate the robots' localization uncertainty, Monte Carlo sampling with multiple cost functions to select the velocities, and the Dynamic Window Approach planner for navigation. The approach is validated both in simulation and in realworld experiments.

Online planning for human-multi-robot interactive theatrical performance (Cappo et al.)

This paper describes a system of aerial robots for improvisational theatrical performances that translates a user's motion specifications into non-colliding and dynamically feasible trajectories. The system is tested in simulation and with up to 15 CrazyFlie quadrotors and showcases the feasibility of real-time multi-robot performances.

On-board communication-based relative localization for collision avoidance in micro air vehicle teams (Coppola et al.)

This paper presents an on-board relative localization method for Micro Air Vehicles (MAVs) that uses only decentralized wireless communication between MAVs. It also describes a reactive collision avoidance algorithm, based on the concept of collision cones, that accounts for the expected performance of the localization method. The proposed strategies for relative localization and collision avoidance are validated both in simulation and in experiments with two types of MAVs.

Robust rendezvous for multi-robot system with random node failures: an optimization approach (Park and Hutchinson)

This paper addresses the rendezvous problem for faulty multi-robot systems. Failures in any robot occur randomly during execution and cannot be detected by its peers. A distributed greedy algorithm is evaluated using numerical simulations, assuming known or worst-case failure probability distributions.

Teleoperation of a platoon of distributed wheeled mobile robots with predictive display (Ha et al.)

This paper presents a distributed framework for teleoperating a platoon of non-holonomic mobile robots through an environment with obstacles. The human operator is shown a map, obtained using SLAM, and the predicted pose of the platoon. The operator provides motion commands to a designated leader robot, which ensures, via local interactions, that the platoon moves in the desired way while retaining its formation.

This has been the second time for an edition of DARS to be followed by a special issue call [the first special issue was (Chirikjian 2014)]. Given the overwhelming response, having received excellent contributions from DARS 2016 and beyond, we are pleased with the outcome, and hope that future editions of the conference will benefit from being associated with a special issue as well.

We would like to acknowledge the excellent work of the authors, reviewers and entire editorial team in helping us to curate this special issue.

\section{References}

Chirikjian, G. (2014). Distributed autonomous robotic systems-Special issue of DARS 2012. Robotica, 32(2), 175.

Groß, R., et al. (Eds.). (2018). Distributed autonomous robotic systems-The 13th international symposium (DARS 2016), In Springer Proceedings in Advanced Robotics (vol. 6).

Publisher's Note Springer Nature remains neutral with regard to jurisdictional claims in published maps and institutional affiliations. 